\title{
Rapid 40 kb genome construction from 52 parts
}

John M. Pryor, Vladimir Potapov, Nilisha Pokhrel, Gregory J. S. Lohman*

Research Department, New England Biolabs, Ipswich, MA, 01938, USA

*To whom correspondence should be addressed. Email: lohman@neb.com

\begin{abstract}
Large DNA constructs $(>10 \mathrm{~kb})$, including small genomes and artificial chromosomes, are invaluable tools for genetic engineering and vaccine development. However, the manufacture of these constructs is laborious. To address this problem, we applied new design insights and modified protocols to Golden Gate assembly. While this methodology is routinely used to assemble 5-10 DNA parts in one-step, we found that optimized assembly permitted >50 DNA fragments to be faithfully assembled in a single reaction. We applied these insights to genome construction, carrying out rapid assembly of the $40 \mathrm{~kb}$ T7 bacteriophage genome from 52 parts and recovering infectious phage particles after cellular transformation. The new Golden Gate assembly protocols and design principles described here can be applied to rapidly engineer a wide variety of large and complex assembly targets.
\end{abstract}

\section{INTRODUCTION}

Large DNA constructs $(\sim 10-50 \mathrm{~kb})$ containing multiple coding regions are widely used in biotechnology for the development of vaccines, therapeutics, and genetically engineered 
organisms. In most cases these constructs are too long to be reliably generated by continuous chemical DNA synthesis methodologies or PCR, and thus are typically assembled from constituent DNA fragments over multiple rounds of assembly [1,2]. Several commonly used methodologies to manufacture large DNA constructs rely on in vivo recombination, and these high capacity systems allow researchers to join up to 40-50 DNA fragments in a single assembly round [3, 4]. However, these techniques typically require lengthy protocols with specialized reagents, and are not amenable to the high-throughput workflows relied upon to engineer desired constructs and gene circuits of a smaller size. In vitro DNA assembly methodologies are not encumbered by the same limitations; however, these methods typically limit researchers to 5-10 DNA fragments per assembly round. Thus, the use of hierarchical assembly schemes that involve multiple rounds of molecular cloning, construct purification, and sequence verification of donor constructs are typical for assembly of large constructs using in vitro assembly methodologies [5-7]. However, recent work by our lab and others suggests that in vitro DNA assembly methods may be able to accommodate many additional fragments per assembly round and thus could be used to rapidly engineer large and complex DNA target sequences [8-10].

Golden Gate assembly (GGA), sometimes referred to as Type IIS assembly, is an in vitro DNA assembly methodology that utilizes a Type IIS restriction endonuclease to generate DNA fragments with short single-stranded overhangs and a DNA ligase to join the fragments together $[11,12]$. This molecular cloning methodology is extremely versatile. Type IIS restriction enzymes cleave outside of their recognition sequence; this allows users to choose the connecting sequences between assembly fragments and has given rise to more than a dozen 'modular' cloning systems with prefabricated parts [6, 13-40]. Additionally, the method can also be used to generate DNA 
targets without the need to introduce unwanted sequence at fusion sites. A significant drawback of GGA is that Type IIS recognition sequences for the enzyme used in the assembly cannot be present in the desired target sequence; however, in most cases this limitation is easily overcome by choosing a Type IIS restriction enzyme with a minimal number of recognition sequences in the desired target and/or introducing silent mutations [41, 42]. Importantly, this method relies on accurate ligation of the single-stranded DNA sequences at fragment fusion sites, as mis-ligation results in improperly ordered fragments and low assembly yield. To avoid erroneous assembly products caused by ligation errors, GGA has typically been limited to $\sim 10$ fragments per reaction; however, a few recent studies have reported $>20$ fragment assembly of constructs up to $10 \mathrm{~kb}[8$, 10, 28]. Recent work by our lab showed that optimization of GGA by data-driven selection of overhang sequences that adjoin assembly fragments, termed Data-optimized Assembly Design (DAD), can significantly expand the limits of this assembly methodology [10].

We have previously shown that using DAD allows for assembly of up to 35 fragments with high fidelity under traditional GGA reaction conditions [10]. Here, to estimate the maximum number of DNA fragments that can be joined in a single GGA reaction, we analyzed the frequency of erroneous fragment assembly under different reaction conditions using a multiplex DNA sequencing assay developed in-house. Our data suggest that $>50$ fragments can be assembled in one reaction with high fidelity using an optimized reaction protocol or with stringent screening conditions, if optimal junction selection rules are followed (Supplementary Text, Figures S1 and $\mathrm{S} 2$, Tables S1 and S2). In the current study we confirmed these predictions in two practical applications: assembly of a $4.9 \mathrm{~kb}$ lac operon cassette into a destination vector from 52 constituent 
fragments, and through assembly of the $40 \mathrm{~kb} \mathrm{T7}$ phage genome from 52 parts in one reaction and $<1$ day.

\section{RESULTS/DISCUSSION}

Our data suggest that prolonged static incubation of GGA reactions at $37^{\circ} \mathrm{C}$ would allow assembly of $>50$ parts with comparable fidelity to our previously reported 35 fragment assembly produced under traditional $37^{\circ} \mathrm{C} / 16^{\circ} \mathrm{C}$ thermocycling conditions (Supplementary Text, Figures S1 and S2, Tables S1 and S2). To test this prediction we sought to carry out the most complex assembly reaction to-date and clone a $4.9 \mathrm{~kb}$ cassette of the lac operon into an E. coli destination vector from 52 constituent parts in a single assembly round (Tables S3-S4). Importantly, the lac operon cassette system used here mimics a traditional cloning reaction wherein, upon transformation of the assembly reaction into $E$. coli cells, we can observe colonies harboring correctly or incorrectly assembled constructs. This test system was engineered to provide a colorimetric readout to differentiate transformants harboring correctly and incorrectly assembled products [8].

DNA fragments comprising the lac operon cassette were generated by PCR and assembled in a reaction containing BsaI-HFv2 and T4 DNA ligase at $37^{\circ} \mathrm{C}$ for 48 hours. Assembly reactions were then transformed into chemically competent $E$. coli cells, and the resulting transformants were scored as having correctly or incorrectly assembled insert sequences. We found that $49 \%$ of the observed transformants harbored correctly assembled constructs, slightly higher than our predicted fidelity of $27 \%$ (Figure 1, Table S3). The reaction generated $>500$ transformants with correctly assembled constructs per $100 \mu \mathrm{L}$ of $E$. coli outgrowth plated, a surprisingly high yield given the complexity of the assembly reaction. To confirm successful assembly of all 52 inserts, constructs 
were purified from a subset of colonies and analyzed by PCR and Sanger sequencing. All constructs from colonies scored as having correct assemblies were found to have inserts of the anticipated size and sequence, and constructs from colonies scored as having incorrect sequences contained truncated inserts (Figure S3). Taken together, these data show that $>50$ fragments can be assembled in one reaction using a high temperature reaction protocol and rational junction selection by DAD.

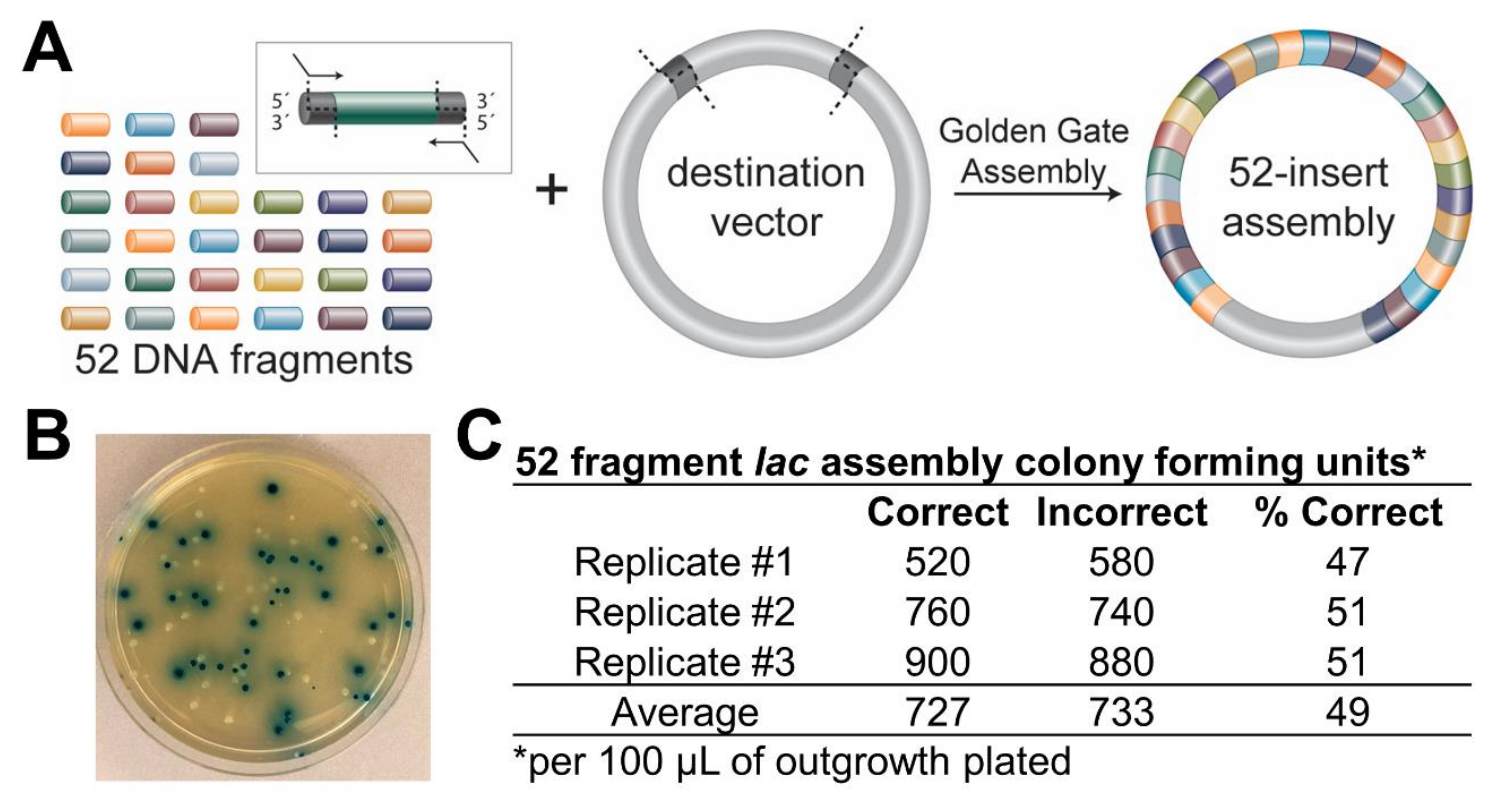

Figure 1. One-pot Golden Gate assembly of 52 fragments into a destination vector.

(A) Schematic of the 52 fragment lac operon cassette assembly. Assembly inserts were generated by PCR amplification and assembled into a destination vector containing an antibiotic resistance marker. (B) Example outgrowth plate used for colorimetric scoring by reverse blue-white screening. Correctly assembled 52 insert constructs form blue colonies upon cellular transformation and incorrectly assembled constructs produce white colonies. (C) Results of the assembly reactions. Three replicate experiments were carried out to quantify the number of colony forming units harboring correct and incorrect assembly products per $100 \mu \mathrm{L}$ of $E$. coli outgrowth plated $(0.2 \mu \mathrm{L}$ of the 
assembly reaction). On average, $49 \%$ of the observed transformants harbored correctly assembled constructs.

Next, we sought to test if using traditional GGA thermocycling protocols would allow for rapid assembly of 50+ fragments, given the use of a more stringent selection system to identify correctly assembled constructs. To test this, we designed a GGA reaction to construct the $40 \mathrm{~kb} \mathrm{~T} 7$ bacteriophage genome from 52 parts (Table S5). Unlike the lac cassette system described above, where circularized constructs with deletions produce colonies due to the presence of the vector antibiotic resistance gene, we reasoned that improperly assembled variants of the $\mathrm{T} 7$ phage genome would contain significant (100s or 1000 s of $n t)$ deletions, and therefore be unlikely to produce viable phage upon cellular transformation. Thus, the $\mathrm{T} 7$ genome results in a more stringent selection where most incorrect assemblies will not form plaques. We reconstructed the phage gDNA from PCR amplicons (Table S4). This strategy enabled us to easily introduce 16 silent mutations to remove pre-existing BsmBI Type IIS restriction sites within the phage genome through PCR primers by choosing break points near all native BsmBI sites. These changes serve dual purpose to both permit Type IIS assembly and to act as marker mutations for assembly verification.

The more stringent selection permitted a shorter, cycled assembly protocol that sacrifices fidelity in favor of efficiency and yield. Assembly reactions were carried overnight using a $42^{\circ} \mathrm{C} / 16^{\circ} \mathrm{C}$ oscillating thermocycling protocol with BsmBI-v2 and T4 DNA ligase. The assembly reactions were then transformed into NEB 10-beta electrocompetent cells; successful assembly of the T7 phage genome was assessed by plaque forming assay. Upon transformation we typically observed 
$\sim 20$ bacteriophage plaques/ $\mu \mathrm{L}$ of assembly reaction, indicating successful assembly of the phage genome (Figure 2). Several phage plaques were selected for additional screening by plaque PCR and restriction enzyme digest to ensure they contained a complete and correctly ordered copy of the T7 phage genome; all plaques subjected to additional screening contained the expected genome arrangement and harbored the intended silent mutations (Figure S4). Moreover, to ensure the observed phage plaques were the result of in vitro assembly and not assembly of the DNA fragments within the E. coli by cellular DNA repair mechanisms, we carried out control reactions lacking T4 DNA ligase and did not observe phage plaques upon transformation of these control reactions. Taken together, these results demonstrate that using a high stringency screen allows rapid assembly of >50 DNA fragments under traditional GGA thermocycling conditions with DAD.
A
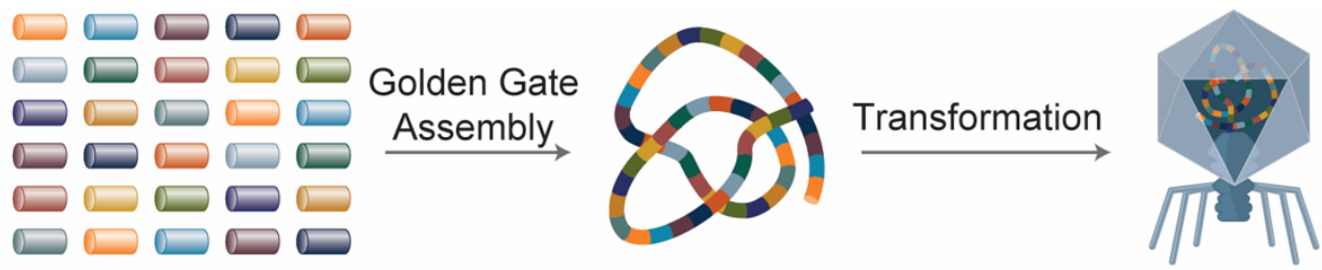
52 DNA fragments
T7 phage gDNA (40 kb)
infectious phage

\section{B 52 fragment T7 gDNA assembly plaque forming units}

\begin{tabular}{cc}
52 fragment 77 gDNA assembly plaque forming units \\
\hline & Plaques* $^{*}$ \\
Replicate \#1 & 46 \\
Replicate \#2 & 6 \\
Replicate \#3 & 23 \\
Replicate \#4 & 18 \\
\hline Average & 23 \\
\hline${ }^{*}$ per $\mu \mathrm{L}$ of assembly reaction &
\end{tabular}

Figure 2: T7 phage genome assembly and infectious phage reconstitution.

(A) Schematic of the 52 fragment T7 phage reconstitution experiment. (B) Results of the assembly reactions. Four replicate experiments were carried out to quantify the 
number of plaque forming units upon transformation of the phage gDNA assembly reactions. On average, 23 plaques were observed per $\mu \mathrm{L}$ of the assembly reaction transformed into $E$. coli cells.

Our results show that $>50$ fragments can be reliably assembled in a single reaction using GGA with DAD. This finding reduces the number of hierarchical assembly rounds required to produce large constructs by in vitro assembly and can be utilized to rapidly assemble entire metabolic pathways and small genomes in a single reaction. Moreover, the large fragment capacity demonstrated here allows for assembly of toxic and/or large DNA constructs from small constituent parts that are easily manipulated and propagated using standard molecular biology techniques, ease of removal of internal Type IIS sites along with fragment generation, and design of large constructs with every ORF on its own fragment. Importantly, this work could facilitate rapid engineering of large DNA targets, as GGA is amenable to automated workflows and supports standardized modular assembly of construct variants with minimal ad-hoc design [43]. In summary, our work demonstrates an efficient and cost-effective means to create and engineer variants of large/complex DNA constructs that are difficult to obtain and manipulate by current cloning and gene synthesis methodologies.

\section{SUPPLMENTARY TEXT}

\section{The impact of reaction temperature on Golden Gate assembly fidelity}

Golden Gate assembly typically utilizes two step cycling protocols, alternating between a $16^{\circ} \mathrm{C}$ incubation step to maximize DNA ligation efficiency and a $37-42^{\circ} \mathrm{C}$ incubation step to maximize fragment digestion efficiency. However, we reasoned that omitting the $16^{\circ} \mathrm{C}$ incubation may 
increase Golden Gate assembly fidelity, as higher reaction temperatures have been shown to improve DNA ligase fidelity [44]. To test this, we carried out a multiplex high throughput DNA sequencing assay developed in-house to quantify the frequency of Golden Gate assembly errors at $37^{\circ} \mathrm{C}$ or $42^{\circ} \mathrm{C}$, and compared the results to reactions using traditional thermocycling protocols of $37 / 16^{\circ} \mathrm{C}$ or $42 / 16^{\circ} \mathrm{C}$ (Figure $\mathrm{S} 1$ ). Of note, the reactions carried out at constant incubation temperatures were incubated for an extended duration of 16 hours to compensate for decreased ligation efficiency. We found that the frequency of ligation errors was reduced $>2$-fold in our sequencing experiments when we omitted the $16^{\circ} \mathrm{C}$ incubation step, with every mismatch pair appearing less frequently. To asses if more fragments could be assembled in one-step by omitting the $16^{\circ} \mathrm{C}$ incubation step during Golden Gate assembly, we next estimated assembly fidelity as a function of fragment number as described previously (Figure S2) [10]. We found that the estimated assembly fidelity for traditional $37 / 16^{\circ} \mathrm{C}$ or $42 / 16^{\circ} \mathrm{C}$ cycling conditions dropped below $10 \%$ at 50 fragments, but the $37^{\circ} \mathrm{C}$ or $42^{\circ} \mathrm{C}$ static incubation protocols could theoretically allow $>50$ fragment to be assembled with $>40 \%$ accuracy. It should be noted that use of the $37^{\circ} \mathrm{C}$ or $42^{\circ} \mathrm{C}$ static temperature protocols is likely to require extended duration reactions to compensate for the ligation efficiency loss caused by omitting the $16^{\circ} \mathrm{C}$ incubation step. Nevertheless, these data suggest that the capacity of Golden Gate assembly reactions could exceed 50 fragments per reaction.

\section{METHODS}

\section{Reagents and Oligonucleotides}

Enzymes, buffers, and media were obtained from New England Biolabs (NEB) unless otherwise noted. Synthetic oligonucleotides were obtained from either Integrated DNA Technologies (IDT) or Sigma Aldrich (Sigma). 


\section{Multiplex DNA Sequencing Assay}

Substrates for the DNA sequencing assay were prepared as previously described [8, 10]. Golden

Gate assembly reactions ( $20 \mu \mathrm{L}$ final volume) to generate sequencing libraries were carried out in 1X T4 DNA ligase buffer by combining: $100 \mathrm{nM}$ of DNA substrate (final concentration) with 2 $\mu \mathrm{L}$ of NEB Golden Gate Enzyme Mix (BsaI-HFv2 or BsmBI-v2). Reactions were carried out for $16 \mathrm{~h}$ at $37^{\circ} \mathrm{C}(\mathrm{BsaI}-\mathrm{HFv} 2)$ or $42^{\circ} \mathrm{C}$ (BsmBI-v2). Reactions were then quenched by the addition of 25 mM EDTA and column purified (Monarch PCR \& DNA Cleanup Kit). The resulting assembly products were further purified to remove un-ligated substrate by treatment with Exonuclease III (50U) and Exonuclease VII (5 U) in 1X Standard Taq Polymerase buffer (final concentration) for $1 \mathrm{~h}$ at $37^{\circ} \mathrm{C}$ in a $50 \mu \mathrm{L}$ reaction volume. The assembly products were then re-purified using the Monarch PCR \& DNA Cleanup Kit and quantified by Agilent Bioanalyzer (DNA 1000).

Pacific Biosciences Single-Molecule Real-Time (SMRT) sequencing was performed as described previously $[8,45]$. The libraries were prepared for sequencing using the PacBio Binding Calculator Version 2.3.1.1 and the DNA/Polymerase Binding Kit P6 v2 with a custom library concentration on the plate of $0.3375 \mathrm{nM}$. Sequencing was carried out using the PacBio RSII instrument with 2 SMRT cells per library and a $3 \mathrm{~h}$ data collection time per cell with 'stage start' off. Consensus sequences for each assembly product were generated as described previously $[8,45]$. Full results from each experiment are supplied in the supporting data files (Tables S1-S2).

\section{Golden Gate Assembly Reactions}

Assembly junction sequences were selected using the optimization described in our previous work [10]. Importantly, these algorithms have been developed into a suite of webtools that can be 
accessed here: https://goldengate.neb.com. Assembly fragments were generated by PCR (Q5 hot start high-fidelity $2 \mathrm{X}$ master mix) with oligonucleotide primers (IDT) and purified using the Monarch PCR \& DNA Cleanup Kit. Fragment quality was evaluated using the Agilent Bioanalyzer 2100 and each assembly part was quantified using the Qubit assay (ThermoFisher). Golden Gate assembly reactions ( $5 \mu \mathrm{L}$ final volume) were carried out with $3 \mathrm{nM}$ of each DNA fragment and $0.5 \mu \mathrm{L}$ of the appropriate NEB Golden Gate Assembly Mix in 1X T4 DNA ligase buffer; the BsaIHFv2 mix was used to reconstitute the lac operon cassette and the BsmBI-v2 mix was used to assemble the $\mathrm{T} 7$ phage genome. Reactions to reconstitute the lac operon cassette were incubated for $48 \mathrm{~h}$ at $37^{\circ} \mathrm{C}$ and then subjected to a final heat-soak step at $60^{\circ} \mathrm{C}$ for 5 minutes before being incubated at $4{ }^{\circ} \mathrm{C}$ prior to transformation. Reactions to produce the $\mathrm{T} 7$ bacteriophage genome were cycled between $42^{\circ} \mathrm{C}$ and $16^{\circ} \mathrm{C}$ for 5 minutes at each temperature for 96 cycles, and then subjected to a $60^{\circ} \mathrm{C}$ incubation for 5 minutes and finally a $4{ }^{\circ} \mathrm{C}$ hold until transformation.

\section{Clonogenic assays}

Assembly reactions to reconstruct the lac operon cassette were transformed into chemically competent E. coli cells, and colony forming units were scored as harboring correctly or erroneously assembled constructs by a reverse blue-white screen as described previously [8, 10]. Briefly, transformations were performed using $2 \mu \mathrm{L}$ of each assembly reaction added to $50 \mu \mathrm{L}$ of T7 express competent cells as per manufacturer's instructions. The resulting outgrowth was plated onto agar plates (Luria-Bertani broth supplemented with $1 \mathrm{mg} / \mathrm{mL}$ dextrose, $1 \mathrm{mg} / \mathrm{mL} \mathrm{MgCl} 2,30$ $\mu \mathrm{g} / \mathrm{mL}$ Chloramphenicol, $200 \mu \mathrm{M}$ IPTG and $80 \mu \mathrm{g} / \mathrm{mL}$ X-gal). Importantly, transformants harboring correctly assembled constructs turn blue after incubation on media containing IPTG and X-Gal, while transformants harboring constructs with assembly errors form white colonies. 


\section{Plaque assays}

Reactions to construct the T7 phage genome were transformed into NEB 10-beta cells as per the manufacturer's instructions, using $1 \mu \mathrm{L}$ of the reaction mixture into $25 \mu \mathrm{L}$ of competent cells. The transfection mixture was recovered in $975 \mu \mathrm{L}$ of NEB 10-beta/stable outgrowth media and then combined with $3 \mathrm{~mL}$ of $50^{\circ} \mathrm{C}$ molten top-agar (Luria broth containing $0.7 \%$ agar). Finally, the mixture was plated on LB agar plates and the molten agar was allowed to cool and solidify on the benchtop for $20 \mathrm{~m}$. The resulting pates were inverted and incubated at $37^{\circ} \mathrm{C}$ for $\sim 5 \mathrm{~h}$ until the $E$. coli lawn and phage plaques were visible by eye.

\section{REFERENCES}

1. W. Zhang, L. A. Mitchell, J. S. Bader, J. D. Boeke, Synthetic Genomes. Annu Rev Biochem 89, 77-101 (2020).

2. A. Casini, M. Storch, G. S. Baldwin, T. Ellis, Bricks and blueprints: methods and standards for DNA assembly. Nat Rev Mol Cell Biol 16, 568-576 (2015).

3. E. D. Postma et al., A supernumerary designer chromosome for modular in vivo pathway assembly in Saccharomyces cerevisiae. bioRxiv doi: 10.1101/2020.02.18.954131 (2020).

4. K. Tsuge et al., Method of preparing an equimolar DNA mixture for one-step DNA assembly of over 50 fragments. Sci Rep 5, 10655 (2015).

5. D. G. Gibson et al., Complete chemical synthesis, assembly, and cloning of a Mycoplasma genitalium genome. Science 319, 1215-1220 (2008).

6. E. Weber, C. Engler, R. Gruetzner, S. Werner, S. Marillonnet, A modular cloning system for standardized assembly of multigene constructs. PLoS One 6, e16765 (2011). 
7. S. Marillonnet, R. Grutzner, Synthetic DNA Assembly Using Golden Gate Cloning and the Hierarchical Modular Cloning Pipeline. Curr Protoc Mol Biol 130, e115 (2020).

8. V. Potapov et al., Comprehensive Profiling of Four Base Overhang Ligation Fidelity by T4 DNA Ligase and Application to DNA Assembly. ACS Synth Biol 7, 2665-2674 (2018).

9. M. HamediRad, S. Weisberg, R. Chao, J. Lian, H. Zhao, Highly Efficient Single-Pot Scarless Golden Gate Assembly. ACS Synth Biol 8, 1047-1054 (2019).

10. J. M. Pryor et al., Enabling one-pot Golden Gate assemblies of unprecedented complexity using data-optimized assembly design. PLoS One 15, e0238592 (2020).

11. C. Engler, R. Kandzia, S. Marillonnet, A one pot, one step, precision cloning method with high throughput capability. PLoS One 3, e3647 (2008).

12. C. Engler, R. Gruetzner, R. Kandzia, S. Marillonnet, Golden gate shuffling: a one-pot DNA shuffling method based on type IIs restriction enzymes. PLoS One 4, e5553 (2009).

13. S. G. Damalas, C. Batianis, M. Martin-Pascual, V. de Lorenzo, V. A. P. Martins Dos Santos, SEVA 3.1: enabling interoperability of DNA assembly among the SEVA, BioBricks and Type IIS restriction enzyme standards. Microb Biotechnol 10.1111/1751-7915.13609 (2020).

14. R. Vasudevan et al., CyanoGate: A Modular Cloning Suite for Engineering Cyanobacteria Based on the Plant MoClo Syntax. Plant Physiol 180, 39-55 (2019).

15. G. M. Taylor, P. M. Mordaka, J. T. Heap, Start-Stop Assembly: a functionally scarless DNA assembly system optimized for metabolic engineering. Nucleic Acids Res 47, e17 (2019).

16. B. Pollak et al., Loop assembly: a simple and open system for recursive fabrication of DNA circuits. New Phytol 222, 628-640 (2019).

17. A. Occhialini et al., MoChlo: A Versatile, Modular Cloning Toolbox for Chloroplast Biotechnology. Plant Physiol 179, 943-957 (2019). 
18. D. Wu, N. Schandry, T. Lahaye, A modular toolbox for Golden-Gate-based plasmid assembly streamlines the generation of Ralstonia solanacearum species complex knockout strains and multi-cassette complementation constructs. Mol Plant Pathol 19, 1511-1522 (2018).

19. C. J. van Dolleweerd et al., MIDAS: A Modular DNA Assembly System for Synthetic Biology. ACS Synth Biol 7, 1018-1029 (2018).

20. D. Lin, C. A. O'Callaghan, MetClo: methylase-assisted hierarchical DNA assembly using a single type IIS restriction enzyme. Nucleic Acids Res 46, e113 (2018).

21. S. P. Leonard et al., Genetic Engineering of Bee Gut Microbiome Bacteria with a Toolkit for Modular Assembly of Broad-Host-Range Plasmids. ACS Synth Biol 7, 1279-1290 (2018).

22. K. Kowarschik, W. Hoehenwarter, S. Marillonnet, M. Trujillo, UbiGate: a synthetic biology toolbox to analyse ubiquitination. New Phytol 217, 1749-1763 (2018).

23. J. Gantner et al., Peripheral infrastructure vectors and an extended set of plant parts for the Modular Cloning system. PLoS One 13, e0197185 (2018).

24. A. I. Andreou, N. Nakayama, Mobius Assembly: A versatile Golden-Gate framework towards universal DNA assembly. PLoS One 13, e0189892 (2018).

25. R. Prielhofer et al., GoldenPiCS: a Golden Gate-derived modular cloning system for applied synthetic biology in the yeast Pichia pastoris. BMC Syst Biol 11, 123 (2017).

26. A. Perez-Gonzalez et al., Adaptation of the GoldenBraid modular cloning system and creation of a toolkit for the expression of heterologous proteins in yeast mitochondria. BMC Biotechnol 17, 80 (2017).

27. U. Obst, T. K. Lu, V. Sieber, A Modular Toolkit for Generating Pichia pastoris Secretion Libraries. ACS Synth Biol 6, 1016-1025 (2017). 
28. A. Martella, M. Matjusaitis, J. Auxillos, S. M. Pollard, Y. Cai, EMMA: An Extensible Mammalian Modular Assembly Toolkit for the Rapid Design and Production of Diverse Expression Vectors. ACS Synth Biol 6, 1380-1392 (2017).

29. S. J. Moore et al., EcoFlex: A Multifunctional MoClo Kit for E. coli Synthetic Biology. ACS Synth Biol 5, 1059-1069 (2016).

30. S. V. Iverson, T. L. Haddock, J. Beal, D. M. Densmore, CIDAR MoClo: Improved MoClo Assembly Standard and New E. coli Part Library Enable Rapid Combinatorial Design for Synthetic and Traditional Biology. ACS Synth Biol 5, 99-103 (2016).

31. N. J. Patron et al., Standards for plant synthetic biology: a common syntax for exchange of DNA parts. New Phytol 208, 13-19 (2015).

32. M. E. Lee, W. C. DeLoache, B. Cervantes, J. E. Dueber, A Highly Characterized Yeast Toolkit for Modular, Multipart Assembly. ACS Synth Biol 4, 975-986 (2015).

33. C. Engler et al., A golden gate modular cloning toolbox for plants. ACS Synth Biol 3, 839843 (2014).

34. Z. Abil, C. A. Denard, H. Zhao, Modular assembly of designer PUF proteins for specific posttranscriptional regulation of endogenous RNA. J Biol Eng 8, 7 (2014).

35. A. Sarrion-Perdigones et al., GoldenBraid 2.0: a comprehensive DNA assembly framework for plant synthetic biology. Plant Physiol 162, 1618-1631 (2013).

36. A. Lampropoulos et al., GreenGate---a novel, versatile, and efficient cloning system for plant transgenesis. PLoS One 8, e83043 (2013).

37. S. Kirchmaier, K. Lust, J. Wittbrodt, Golden GATEway cloning--a combinatorial approach to generate fusion and recombination constructs. PLoS One 8, e76117 (2013). 
38. S. Werner, C. Engler, E. Weber, R. Gruetzner, S. Marillonnet, Fast track assembly of multigene constructs using Golden Gate cloning and the MoClo system. Bioeng Bugs 3, 3843 (2012).

39. A. Sarrion-Perdigones et al., GoldenBraid: an iterative cloning system for standardized assembly of reusable genetic modules. PLoS One 6, e21622 (2011).

40. W. Szybalski, S. C. Kim, N. Hasan, A. J. Podhajska, Class-IIS restriction enzymes--a review. Gene 100, 13-26 (1991).

41. P. Pullmann et al., Golden Mutagenesis: An efficient multi-site-saturation mutagenesis approach by Golden Gate cloning with automated primer design. Sci Rep 9, 10932 (2019).

42. Z. Zhang, K. Xu, Y. Xin, Z. Zhang, An efficient method for multiple site-directed mutagenesis using type IIs restriction enzymes. Anal Biochem 476, 26-28 (2015).

43. L. Ortiz, M. Pavan, L. McCarthy, J. Timmons, D. M. Densmore, Automated Robotic Liquid Handling Assembly of Modular DNA Devices. J Vis Exp 10.3791/54703 (2017).

44. G. J. Lohman et al., A high-throughput assay for the comprehensive profiling of DNA ligase fidelity. Nucleic Acids Res 44, e14 (2016).

45. V. Potapov et al., A single-molecule sequencing assay for the comprehensive profiling of T4 DNA ligase fidelity and bias during DNA end-joining. Nucleic Acids Res 46, e79 (2018).

\section{ACKNOWLEDGEMENTS}

We thank Tasha José (New England Biolabs) for providing illustrations as well as and Rebecca Kucera and Eric Cantor (New England Biolabs) for providing reagents. We also thank Katharina Bilotti, Na Ke, Rebecca Kucera, and Eric Cantor (New England Biolabs) for careful reading of the manuscript. 
bioRxiv preprint doi: https://doi.org/10.1101/2020.12.22.424019; this version posted December 22, 2020. The copyright holder for this preprint (which was not certified by peer review) is the author/funder, who has granted bioRxiv a license to display the preprint in perpetuity. It is made available under aCC-BY-ND 4.0 International license. 


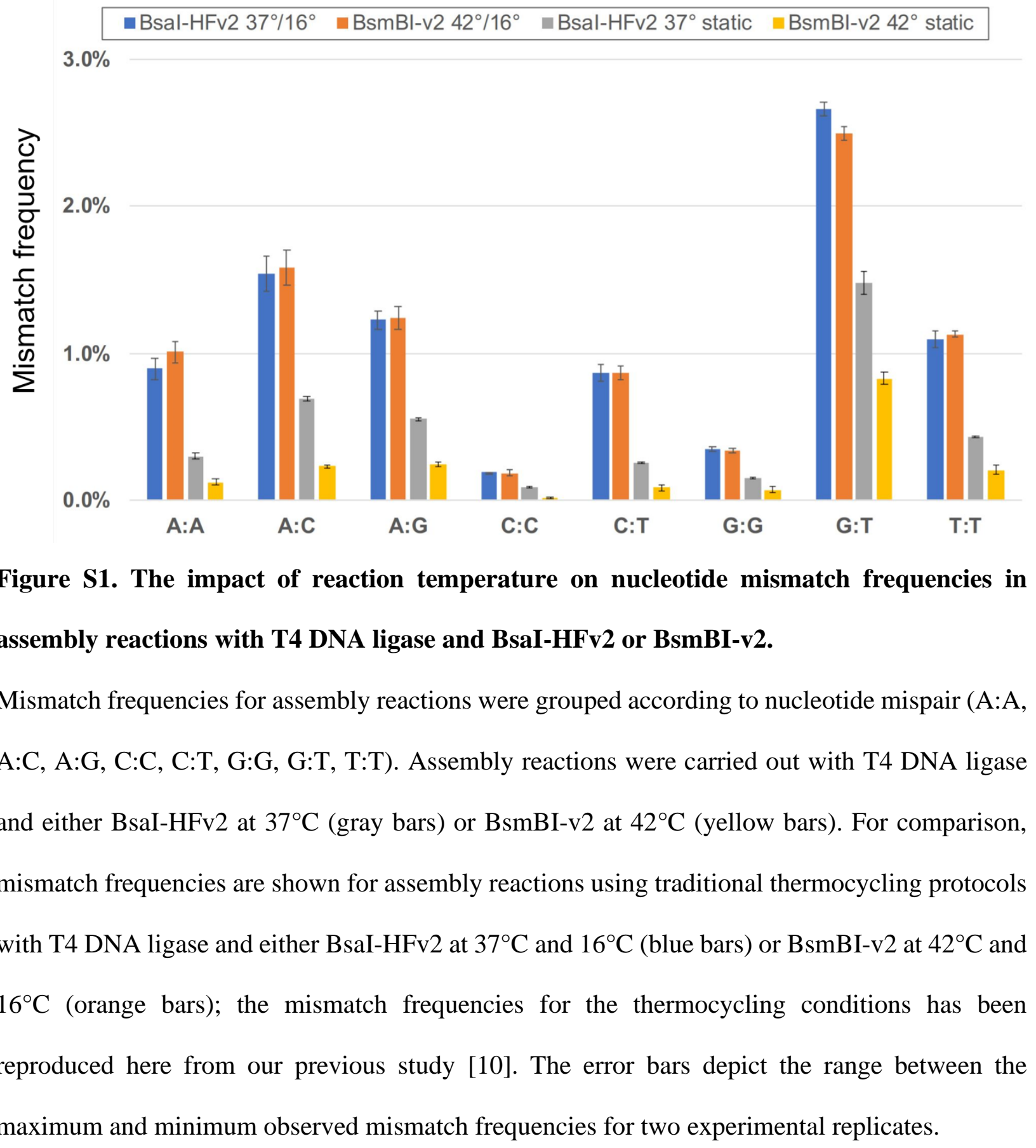




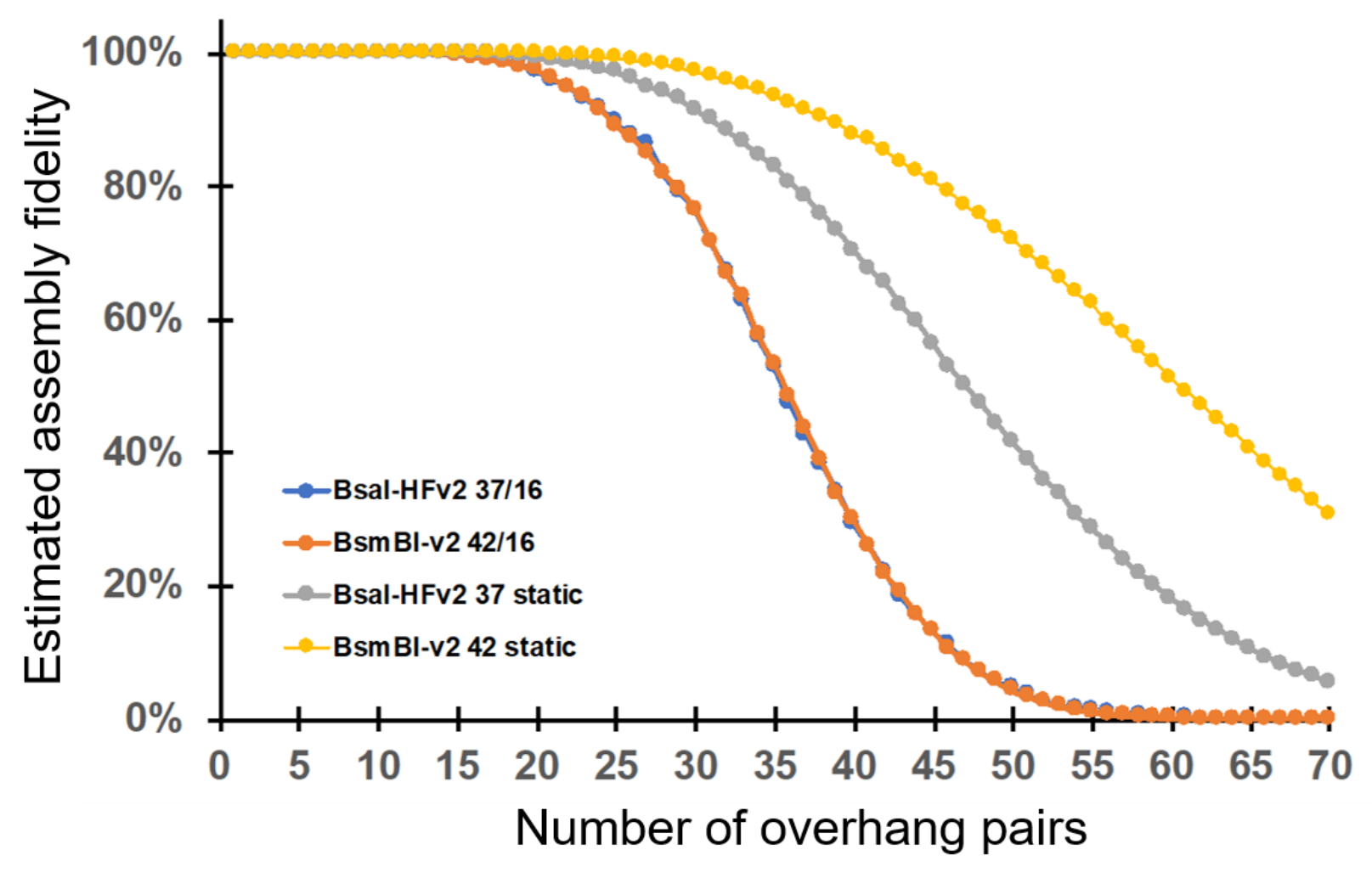

Figure S2. Golden Gate assembly fidelity predictions as a function of the overhang pairs in the assembly reaction.

Fidelity estimations were carried out using the data from the multiplex DNA sequencing assay as described previously [10]. The estimated assembly fidelity of reactions containing up to 70 overhang pairs is shown for reactions using thermocycling conditions with T4 DNA ligase and BsaI-HFv2 (blue) or BsmBI-v2 (orange), or with T4 DNA ligase and BsaI-HFv2 at $37^{\circ} \mathrm{C}$ (gray) or BsmBI-v2 at $42^{\circ} \mathrm{C}$ (yellow). 


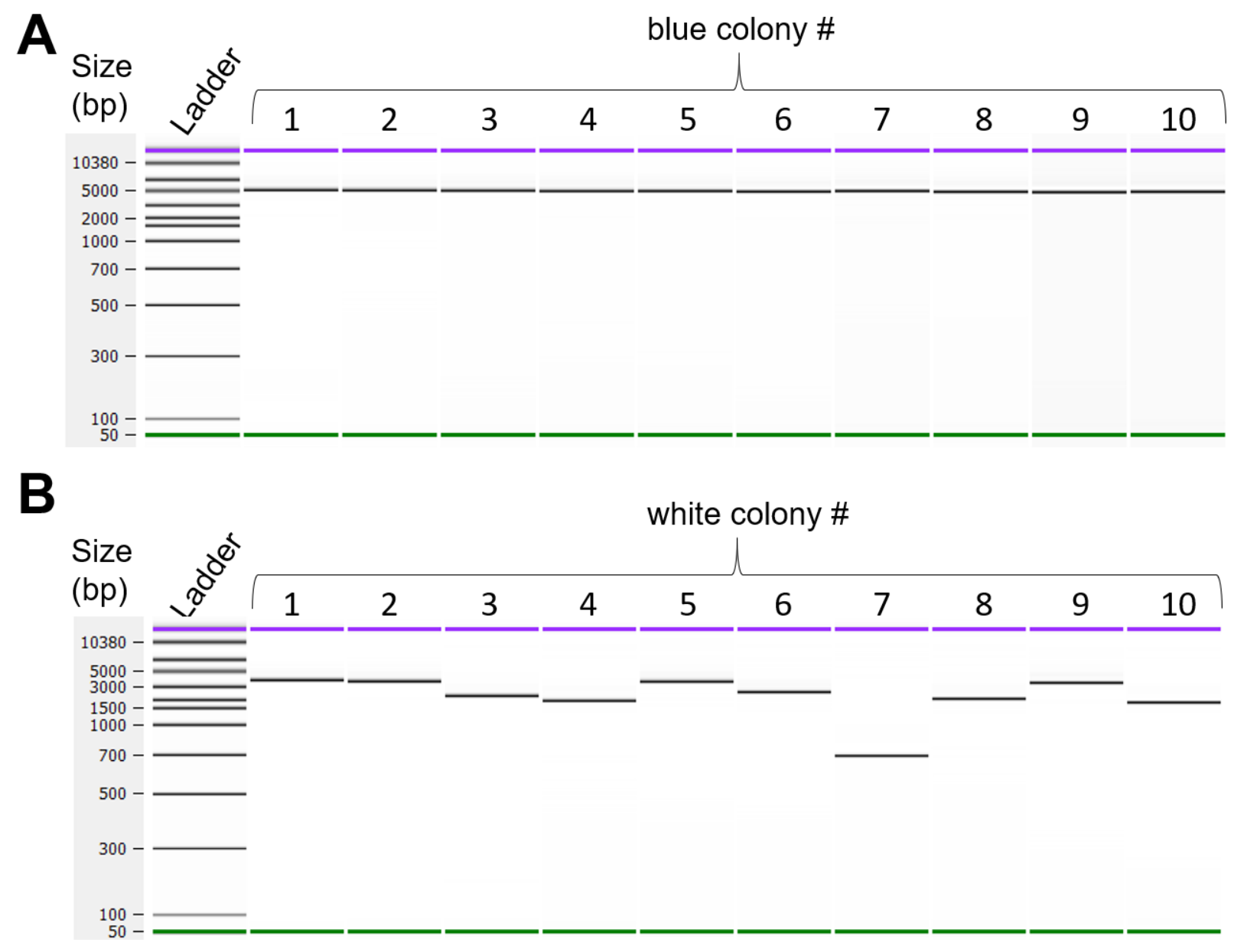

Figure S3. Verification of the 52 fragment lac operon cassette assembly.

Plasmid DNA was isolated from colonies using the Monarch Plasmid Miniprep kit and subjected to PCR with amplification primers that flank the desired insertion site. As anticipated, (A) blue colonies contained inserts of the expected size for correct assembly of all 52 fragments, and (B) white colonies harbored constructs carrying truncated assembly products. 


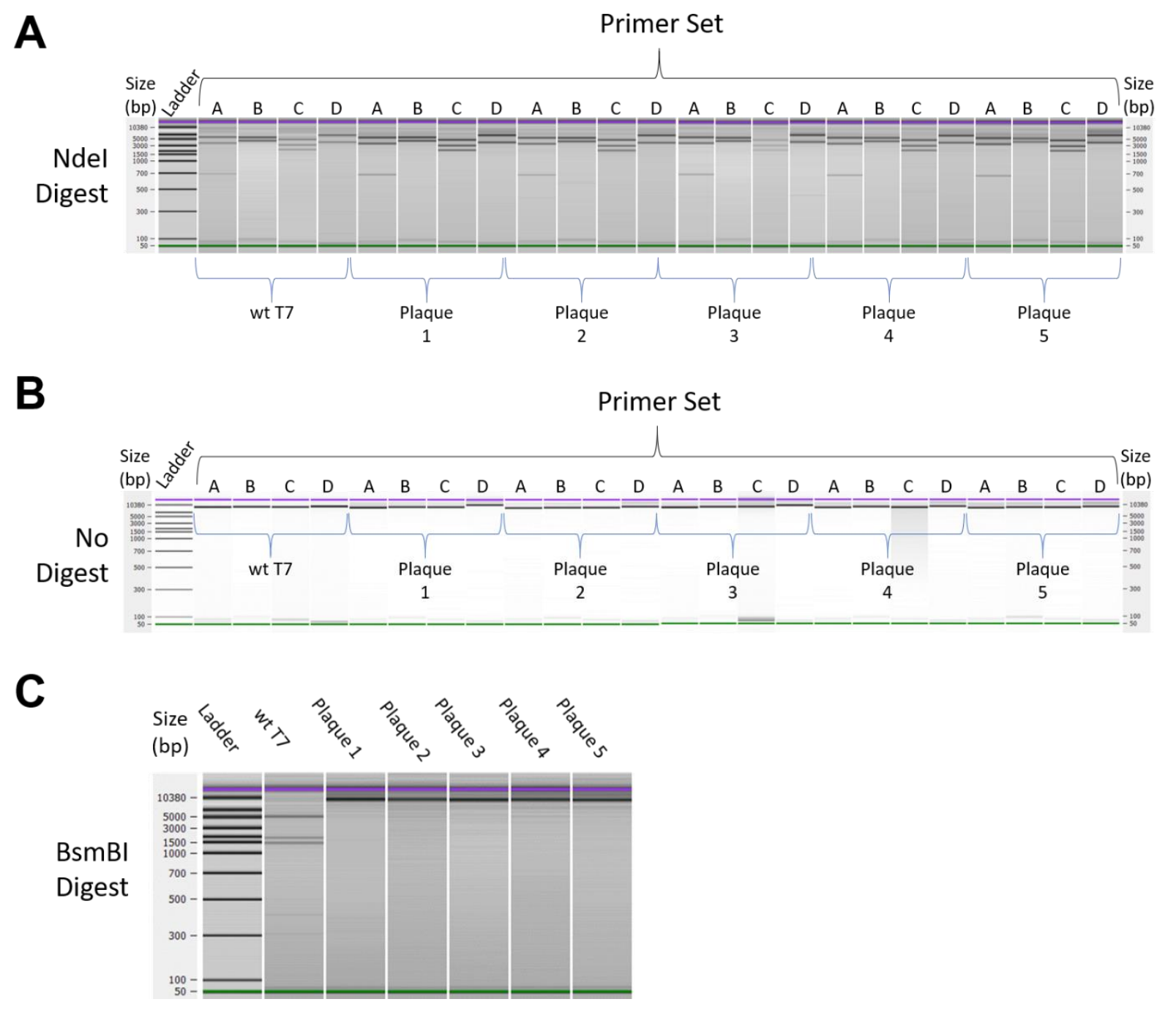

\section{Figure S4. Verification of the 52 fragment T7 phage gDNA assembly.}

Plaque PCR was carried out using 4 sets of amplification primers (A-D) that together span the 40 kb phage genome. Amplicon lengths were resolved by Agilient Bioanalzyer 2100, using a DNA 12000 assay. Amplicons from 5 phage plaques were compared to the parental wt T7 phage genome after restriction enzyme digest with $\mathrm{NdeI}(\mathrm{A})$ or undigested (B). In all cases, the phage plaques produced a pattern identical to the parental wt T7 gDNA. (C) To confirm that the assembled genomes harbored the desired silent mutations to remove native BsmBI restriction sites, we also carried out amplicon digestion with BsmBI. We show that amplicon D from the parental T7 phage 
genome is digested by BsmBI, whereas amplicon D from the assembled phage genomes is inert to cleavage by BsmBI.

Table S1. Ligation frequency for each overhang pair in assembly reactions with BsaI-HFv2 and T4 DNA ligase at $37^{\circ} \mathrm{C}$

Table S2. Ligation frequency for each overhang pair in assembly reactions with BsmBI-v2 and T4 DNA ligase at $42^{\circ} \mathrm{C}$

Table S3. 52 fragment lac operon cassette assembly fragments and overhang sequences

Table S4. Primer sequences

Table S5. 52 fragment T7 phage assembly fragments and overhang sequences 